Trauma Berufskrankh 2015 • [Suppl 2]:

17:281-282

DOI 10.1007/s10039-015-0060-2

Online publiziert: 25. Juni 2015

๑) Springer-Verlag Berlin Heidelberg 2015

CrossMark

Die Haupttätigkeiten des niedergelassenen Durchgangsarztes im operativen Bereich umfasst die notfallmäßige unvorhergesehene Wund- und Frakturbehandlung sowie das ambulante Operieren von gesetzlich Unfallversicherten nach Anhang 2 EBM und $\$ 115$ b SGB V.

\section{Fachliche und räumliche Veränderungen}

Die Neuordnung der berufsgenossenschaftlichen Heilverfahren 2011 hat für den niedergelassenen D-Arzt viele Veränderungen auch in der operativen Tätigkeit gebracht.

Der neue D-Arzt muss zum Führen der deutschen Facharztbezeichnung „Orthopädie und Unfallchirurgie“ berechtigt sein und zusätzlich die Bezeichnung „spezielle Unfallchirurgie“ nachweisen, wenn er zulasten der gesetzlichen Unfallversicherung uneingeschränkt ambulant operieren will. Ansonsten verbleibt ein nur begrenzter Katalog von 16 kleineren operativen Eingriffen.

Unabhängig davon müssen jedoch als räumliche Voraussetzungen 2 Räume für invasive Eingriffe, getrennt nach Kontaminationsgrad, bereitgehalten werden, wobei einer der Räume die Kriterien nach $\$ 115$ b SGB V bei entsprechender Nutzung erfüllen muss. Diese Raumkonditionen entsprechen nach Meinung des Autors nicht mehr aktuellen Hygienevorstellungen.

Außerdem ist der D-Arzt nach $\$ 12$ Vertrag Ärzte/Unfallversicherungsträger berechtigt, Arbeitsunfallverletzte an Ärzte, die zum ambulanten Operieren in der vertragsärztlichen Versorgung berechtigt sind, zur ambulanten Leistungserbringung, auch ambulanten Operation, zu überweisen. In der vertragsärztlichen Versorgung ist bei Durchführung ambulanter

\title{
C. Schüürmann
}

1. Vors. Berufsverband niedergelassener Chirurgen (BNC), Bad Homburg, Deutschland

\section{Ambulantes Operieren in der gesetzlichen Unfallversicherung}

Operationen auch nach $\$ 115 \mathrm{~b}$ SGB V eine 2-Raum-Lösung nicht gefordert.

Dazu kommen die allseits bekannten verschärften Hygieneverordnungen, die spätestens seit $2012 \mathrm{zu}$ einer deutlichen Kostenzunahme durch sächliche und personelle Aufrüstung gerade beim ambulanten Operieren geführt haben. Dies schließt auch die zusätzlichen Begehungen durch bisher viel zu wenige Krankenhaushygieniker sowie Investitionen für z. T. von Gesundheitsämtern geforderten raumlufttechnischen Anlagen ein. Allerdings gibt es bisher keinen überzeugenden Beweis (außer sehr geringfügig z. B. bei Hüftprothesenimplantationen) für einen gesicherten hygienischen Mehrnutzen.

Weiterhin ist kritisch anzumerken, dass in der Kalkulation einer ambulanten Operationseinheit, die auch für den Unfallversicherten bereitgehalten wird, der in Deutschland übliche Produktivitätsindex von $87,5 \%$ gilt, der ursprünglich um 1990 in der Schweiz erhoben wurde und dort als Wert (85\%) für die Kostenstelle Arztzimmer zugrunde gelegt wurde. Was dies mit einem Operationsraum und dessen Betriebskosten von heute zu tun haben soll, ist keinem Kollegen mehr vermittelbar, insbesondere angesichts der beschriebenen geänderten Hygienevorschriften, die nunmehr Krankenhausniveau voraussetzen. Der Wert $87,5 \%$ bedeutet, dass $12,5 \%$ auf die Schnitt-NahtZeit für die Preisgestaltung hinzugerechnet werden!

Allgemein, also auch für bereits bestallte D-Ärzte, wurde die Verpflichtung zur D-ärztlichen regelmäßigen Fortbildung erweitert und in einem sich wiederholenden 5-Jahres-Zeitraum inhaltlich festgelegt mit Teilnahme an 2 Tagungen der DGUV-Landesverbände sowie Kindertraumatologie, Begutachtungswe- sen, Reha-Management und Reha-Medizin. Letzteres steht als Fortbildungsangebot erst seit Kurzem überhaupt nennenswert zur Verfügung.

Der neue D-Arzt wird also in Zukunft mindestens 8 Jahre Weiterbildung benötigen, wenn er erfolgreich die Kammerurkunde Röntgendiagnostik Skelett während der Facharztweiterbildung erworben hat, ansonsten muss er 18 Monate in einer radiologischen Abteilung verlängern.

Nach Einschätzung des BNC benötigt der neue D-Arzt in der Niederlassung zusätzlich den Facharzt für Allgemeinchirurgie, wenn er „nur“ die formalen fachlichen Voraussetzungen der jetzigen Praxisinhaber als Nachfolger erfüllen will, das bedeutet eine Mindestweiterbildungszeit von 11 Jahren, was eine Zumutung darstellt.

\section{Veränderungen zur Vergütung}

Auf der anderen Seite hat es verschiedene Veränderungen in der Vergütung gegeben, seit 2005 gibt es deutliche Zuschläge zum ambulanten Operieren, seit 2012 ebenso deutliche Aufwertungen häufig vorkommender operativer Eingriffe durch Beschlüsse der ständigen Gebührenkommission, teilweise mit erhöhtem dokumentatorischen Aufwand.

Dazu wurden und werden gerade die Vergütungen der verschiedenen Gutachtenformen der berufsgenossenschaftlichen Berichterstattung deutlich erhöht, allerdings unter Anpassung der dazugehörenden Leistungslegenden.

Inzwischen haben in den Bundesländern unter Beteiligung und Anschubfinanzierung des Berufsverbands der Deutschen Chirurgen Clearingstellen ihre Arbeit aufgenommen, um Differenzen bei den Rechnungsstellungen zwischen 
Ärzten und Unfallversicherungsträgern zu klären und beizulegen.

\section{Schlussfolgerung}

Zusammenfassend bestehen durch die Neuordnung des berufsgenossenschaftlichen Heilverfahrens erhöhte persönliche fachliche Voraussetzungen und vermehrte Kosten durch insgesamt geforderte zusätzliche bauliche, sächliche und personelle Ausstattung sowie eine teilweise Anpassung der Gebühren sowie weiterhin Einzelleistungsvergütung.

Dennoch wird die uneingeschränkte Forderung aufrechterhalten, dass reale Kosten gerade im operativen Bereich auch in der gesetzlichen Unfallversicherung durch anerkannte moderne Erhebungsverfahren neu ermittelt werden einschließlich aller anfallenden Overheadleistungen und dass Kosten grundsätzlich nicht mehr mit dem Arztlohn verrechenbar sein dürfen.

\section{Korrespondenzadresse}

\section{Dr. C. Schüürmann}

1. Vors. Berufsverband niedergelassener

Chirurgen (BNC), Louisenstr. 53-57

61348 Bad Homburg

C.Schueuermann@gmx.de

\section{Einhaltung ethischer Richtlinien}

Interessenkonflikt. C. Schüürmann gibt an, dass kein Interessenkonflikt besteht.

Dieser Beitrag beinhaltet keine Studien an Menschen oder Tieren.

The supplement containing this article is not sponsored by industry.

Trauma Berufskrankh 2015 • [Suppl 2]: 17:281-282 DOI 10.1007/s10039-015-0060-2

(c) Springer-Verlag Berlin Heidelberg 2015

\section{Schüürmann}

\section{Ambulantes Operieren in der gesetzlichen Unfallversicherung}

Zusammenfassung

Hintergrund. Die Neuordnung der berufsgenossenschaftlichen Heilverfahren hat auch für den niedergelassenen Durchgangsarzt viele Veränderungen gebracht.

Fachliche und räumliche Veränderungen. Die fachlichen Voraussetzungen wurden deutlich verändert, die räumlichen Voraussetzungen bleiben erhalten bzw. sind durch begleitende Hygieneverordnungen verschärft worden. Die Verpflichtung zur regelmäßigen Fortbildung als bestallter D-Arzt wurde präzisiert und ausgeweitet. Weiter benötigt der neue D-Arzt mindestens 8 Jahre Weiterbildung; will er die formalen Qualifikationen der meisten heutigen Praxisinhaber erreichen, sind es mindestens 11 Jahre (Doppelfacharzt).

Änderungen in der Vergütung. Zu nennen sind seit 2005 Zuschläge zum ambulanten Operieren, seit 2012 Aufwertungen häufig vorkommender Eingriffe, teilweise mit erhöhtem dokumentatorischen Aufwand, sowie bei Anpassung der Leistungslegenden eine Erhöhung der Gutachtengebühren. Zur Lösung von Differenzen in der Rechnungsstellung haben inzwischen Clearingstellen ihre Arbeit aufgenommen.

Schlussfolgerung. Es bleibt die Forderung, endlich reale Kosten im operativen Bereich durch anerkannte moderne Erhebungsverfahren zu ermitteln und einschließlich der Overheadleistungen auch in der gesetzlichen Unfallversicherung vollständig zu vergüten.

Schlüsselwörter

Ambulante Versorgung · Niedergelassener

D-Arzt · Berufsgenossenschaftliches

Heilverfahren · Fortbildung ·

Gesundheitskosten

\section{Outpatient surgery in the statutory accident insurance}

\section{Abstract}

Background. The rearrangement of the medical procedure of the occupational mutual insurance scheme has also brought many changes for registered practitioners as accident insurance consultants ( $D$-Arzt).

Professional and situational alterations. The professional qualifications have been markedly altered but the layout conditions have remained unaltered or have become intensified by accompanying hygiene regulations. The obligation to undertake regular further training as an appointed D-Arzt has been defined more precisely and broadened. Furthermore, a newly appointed $D$-Arzt must undertake at least 8 years further training and to achieve the formal qualifications of most currently practicing $D$-Ärzte, at least 11 years (double medical specialist).

Amendments in remuneration. Noteworthy are that supplementary charges for out- patient surgery were introduced in 2005, upgrading of frequently performed interventions in 2012, sometimes with increased workload for documentation and an increase in the expert opinion fees for adaptation of the service legends. In order to solve the differences in the invoicing, clearing houses have now been installed.

Conclusion. There is still a need to finally determine the real costs in the operative field by recognized modern methods of data collection and including a complete remuneration of the overheads incurred even in the statutory accident insurance.

Keywords

Ambulatory care $\cdot$ Accident insurance consultant - Occupational mutual insurance association · Education $\cdot$ Health care costs 